\title{
PEPTIDOGLYCAN FROM Staphylococcus aureus INDUCES THE OVEREXPRESSION OF TRLS 1-8 mRNA IN CORNEAL FIBROBLASTS, BUT ITS LIPOTEICHOIC ACID AND MURAMYL DIPEPTIDE ONLY INDUCED THE OVEREXPRESSION OF TLR5 OR TRL9
}

\author{
Sandra Rodríguez-Martínez ${ }^{1}$, Norma A. Sánchez-Zauco ${ }^{1}$ Ivonne González-Ramírez², Juan C. Cancino-Diaz ${ }^{2}$, Mario E. \\ Cancino-Diaz ${ }^{1^{*}}$
}

${ }^{1}$ Department of Immunology, Escuela Nacional de Ciencias Biológicas-Instituto Politécnico Nacional, Mexico City, Mexico; ${ }^{2}$ Department of Microbiology, Escuela Nacional de Ciencias Biológicas-Instituto Politécnico Nacional, Mexico City, Mexico.

Submitted: July 12, 2010; Returned to authors for corrections: December 27, 2010; Approved: March 14, 2011.

\begin{abstract}
Lipopolysaccharide induces TLR-1-8 mRNAs over-expression in corneal fibroblast. Analyzing if other TLR-ligands can do the same, we found that peptidoglycan does, but not muramyldipeptide, lipoteichoic acid and polyI:C. This suggests that the recognition of lipopolysaccharide and peptidoglycan is enough to alert these cells against microorganisms through the over-expression of the majority TLRs.
\end{abstract}

Key words: peptidoglycan, TLRs, corneal fibroblasts.

It has been described that Toll Like Receptors (TLRs) recognize a specific Pathogen-Associated Molecular Pattern (PAMP). For instance, TLR-4 recognizes lipopolysaccharide (LPS), TLR-9 recognizes bacterial unmethylated DNA, and TLR-3 and TLR-7 are activated by double- and single-stranded RNAs, respectively (1). However, TLR-2 recognizes a variety of PAMPs such as peptidoglycan (PGN), lipoteichoic acid (LTA), lipopeptides, zymozan, among others (1). The expression of TLRs can be constitutive or induced. It is well known that a specific PAMP can induce the over-expression of its specific TLR, and the most well studied example is LPS, which can over-express TLR-4 (4). However, this overexpression is not exclusive of a specific TLR, since it has been observed that the induction with a particular PAMP can induce the over-expression of several TLRs, for instance, LPS can increase the expression of other TLRs not related to LPS such as TLR-2, $-3,-6$ and -7 in primary human intestinal myofibroblast cells (9). Our group has previously reported that murine Balb/c corneal fibroblast cells treated with LPS from Escherichia coli over-express TLRs from 1 to 9, and that murine NIH corneal fibroblasts cells over-express TLRs from 1 to 7 using the same stimuli (10). In order to keep exploring the behavior of corneal fibroblasts when they are treated with different PAMPs, we worked with PGN and LTA from Staphylococcus aureus, recognized by extracellular Pathogen Recognition Receptors (PRRs), and muramyldipeptide (MDP) and synthetic poly I:C, recognized by intracellular PRRs, to assess the ability of these cells to increase the expression of TLRs mRNAs.

Corneal fibroblast cultures were prepared according to

*Corresponding Author. Mailing address: Carpio y Plan de Ayala S/N, Col. Santo Tomas, Delegación Miguel Hidalgo, 11340, Mexico City, Mexico.; Tel.: 5557296000 Ext: 46209.; E-mail: jccancinodiaz@ hotmail.com 
Rodríguez-Martínez et al (10). Mice were manipulated following the guidelines of the Research Committees of the Instituto Politécnico Nacional. Briefly, ICR mice's corneas were digested with $24 \mathrm{U}$ dispase solution (Invitrogen, Carlsbad, CA, USA) at $37^{\circ} \mathrm{C}$ for 4 hours, followed by 10 minutes of digestion with trypsin-EDTA (Invitrogen) at $37^{\circ} \mathrm{C}$. After washes with DMEM (Invitrogen), the cells were resuspended in DMEM $(150,000$ cells $/ \mathrm{mL}$ ) containing $5 \%$ of fetal bovine serum (Gibco, Rockwille, MD, USA), gentamicin, and fungizone and the cells were cultured overnight. The corneal fibroblasts, cultured in six well plates, (90\% of confluence) were treated with PGN $(10 \mu \mathrm{g} / \mathrm{mL})$, LTA $(10$ $\mu \mathrm{g} / \mathrm{mL}), \operatorname{MDP}(10 \mu \mathrm{g} / \mathrm{mL})$ from Staphylococcus aureus (Sigma, St. Louis, MO, USA) and with poly I:C (7 $\mu \mathrm{g} / \mathrm{mL}$; Amersham Biosciences, Westborough, MA, USA) in fresh DMEM medium for 3, 6 and 12 hours. After treatment the cellular viability was assessed with trypan blue, and not less than $92 \%$ was observed. Total RNA extraction was performed with the TRIzol (Invitrogen) reagent from treated cultures. Total RNA was treated with DNAse I (Invitrogen) and RNA was re-extracted with TRIzol, and RTPCR to TLRs and to glyceraldehyde 3-phosphate dehydrogenase
(GAPDH) were done according to Rodríguez-Martínez et al (10).

In Figure 1 it can be clearly observed that PGN is the most potent inducer used for TLRs over-expression. After 3 hours of treatment with PGN, TLR-1, TLR-6, and TLR-2 mRNAs, which are specific receptors for this PAMP, were over-expressed, as well as TLRs that are not specific to this PAMP (TLR-3, TLR-4, TLR5, TLR-7 and TLR-8 mRNAs; Figure 1, panel A). TLR-9 was not over-expressed after this stimulus. This result is in accordance with those found by Rodriguez-Martinez et al (10) and Otte et al (9) who documented that murine corneal fibroblasts and intestinal myofibroblasts, respectively, treated with LPS induce the overexpression of the majority of TLRs mRNAs. Based on these results we suggest that the recognition of one PAMP is enough to induce the cell to over-express all TLRs mRNAs to fight a probable infection against several kinds of microorganisms. These results are interesting because the main role of fibroblasts has been thought to be the production of collagen to give support, elasticity and strength to the corneal tissue, and here we show that these cells can also participate in the defense against microorganisms through the over-expression of TLRs mRNA.
A

PGN

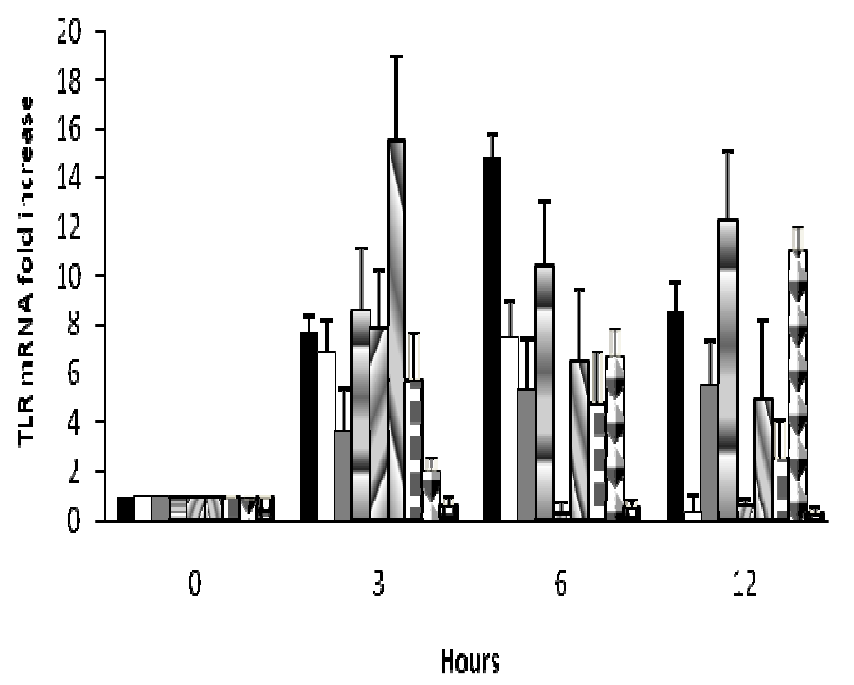

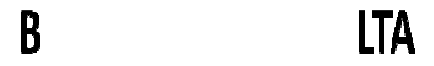

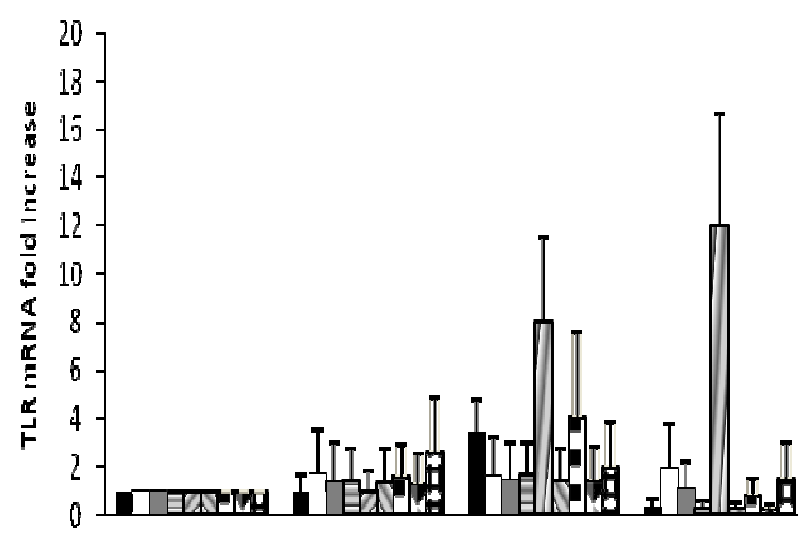

0

3

6

12

Hours 

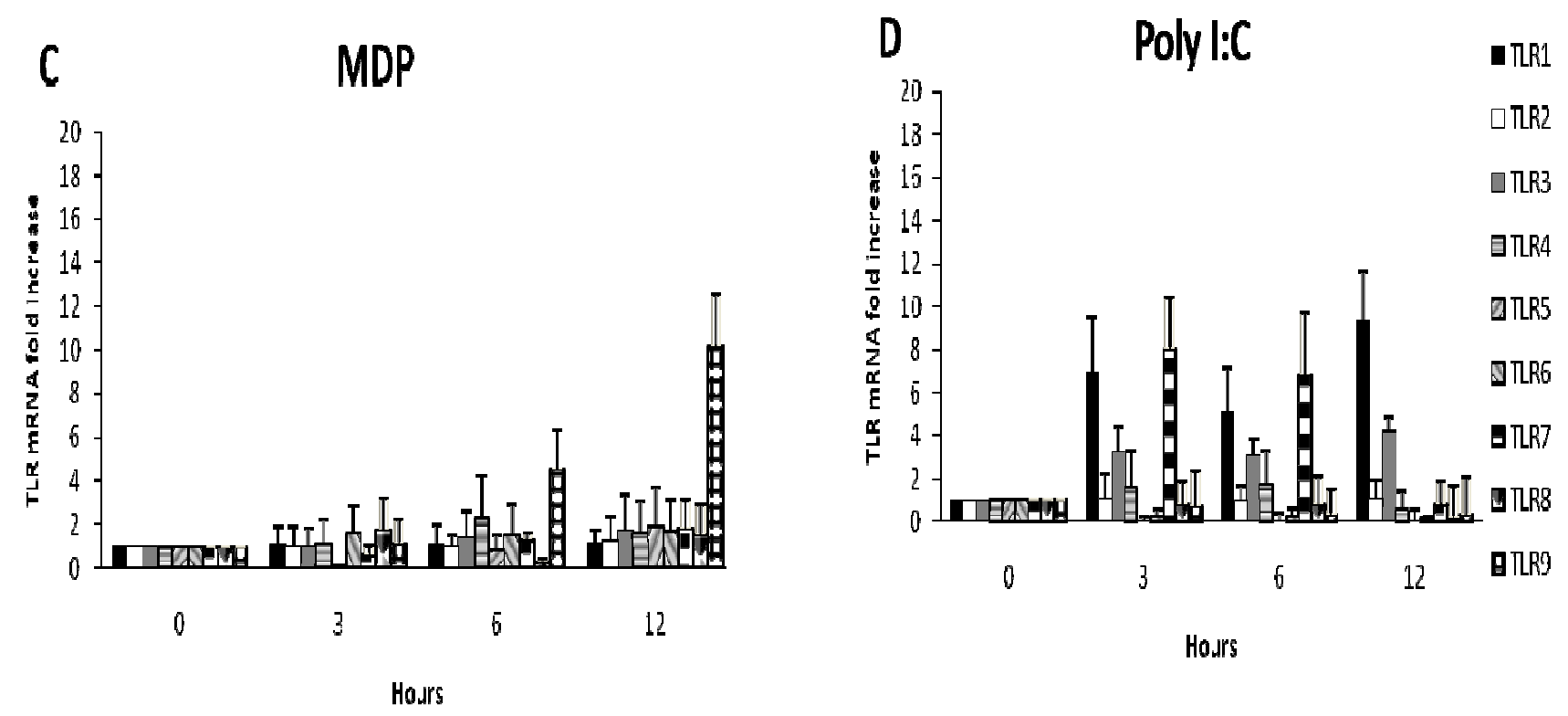

Figure 1. TLR mRNA fold increase in corneal fibroblasts treated with cell wall components from Staphylococcus aureus. Corneal fibroblasts were treated with PAMPs for 3, 6 and 12 hours. Panel A: peptidoglycan (PGN), $10 \mu \mathrm{g} / \mathrm{mL}$; Panel B: lipoteichoic acid (LTA), $10 \mu \mathrm{g} / \mathrm{mL}$; Panel C: muramyldipeptide (MDP), $10 \mu \mathrm{g} / \mathrm{mL}$; Panel D: poly I:C, $7 \mu \mathrm{g} / \mathrm{mL}$. Semiquantitative RT-PCR was done to determinate the expression of TLRs mRNAs. TLRs mRNA fold expression was calculated as follows: intensity of amplicon bands was analyzed with Alpha Imager software. The band intensities were normalized with the corresponding GAPDH signal (TLR/GAPDH rate) and this value was normalized with the corresponding control (0 hours). Data were analyzed by the Kruskal-Wallis statistical test.

LTA is another cell wall component of $S$. aureus bacterium that is recognized by the heterodimer formed by TLR-2/TLR-1 (13). When corneal fibroblast cells were stimulated with LTA, only TLR-5 mRNA was over-expressed at 6 and 12 hours after treatment (Figure 1, panel B). Again, with this PAMP, TLR-9 mRNA was not over-expressed. In contrast to PGN, LTA did not induce the over-expression of its specific TLR-2 and TLR-1 mRNAs. This discrepancy of response between PGN and LTA in these cells could be attributable to differences on signaling pathways induced by specific PAMPs, since it has been demonstrated that LTA from $S$. aureus can induce the extracellular-signal-regulated kinases (ERK) signaling pathway in corneal fibroblasts, but not the nuclear factor kappa-light-chain-enhancer of activated B cells $(\mathrm{NF}-\mathrm{\kappa B})$ signaling pathway, while tumor necrosis factor (TNFalpha) in the same cells activates this transcription factor (14). In the same way PGN, but not LTA, stimulates the activation of NF- $\mathrm{KB}$ in corneal epithelial cells (7), and in lung epithelial cells PGN, but not LTA, induces the production of interleukin8 (IL-8) and granulocyte-macrophage colony-stimulating factor (GM-CSF) (5). In contrast, LTA can stimulate human betadefensin 2 expression in a NF- $\mathrm{BB} / \mathrm{TLR}$-dependent pathway in human airway epithelial cells (11). Therefore, it seems that the ability of cells to recognize PGN and LTA is tissue-specific and it could be because heterodimer TLR-2/TLR-1 is not expressed constitutively in LTA non-responsive cells (8). 
MDP was able to induce the over-expression of TLR-9 mRNA after 6 and 12 hours of treatment. The other TLRs were not over-expressed (Figure 1, panel C). MDP is a PGN degradation product of enzymes with $\mathrm{N}$-acetylmuramoyl-L-alanine amidase activity, such as peptidoglycan receptor protein-2 (3). It is internalized into the cells and is recognized by the intracellular nucleotide-binding oligomerization domain containing 2 (NOD2) receptor. We found that when corneal fibroblasts were treated with MDP, only TLR9 (an intracellular TLR) was over-expressed. It is important to highlight that PGN induces all TLRs except TLR-9, which in turn is inducible by MDP, hence PGN and MDP together lead to the induction of all TLRs. It has been demonstrated that NOD1 and NOD2 mRNAs are expressed in corneal fibroblasts (10) and that MDP can increase the expression of these molecules (personal communication) indicating that corneal fibroblasts can recognize intracellular PAMPs.

In order to analyze if corneal fibroblasts can also respond to a non-bacterial PAMP, these cells were treated with poly I:C (considered as dsRNA and recognized by intracellular TLR-3), and they over-expressed TLR-1, TLR-3, and TLR-7 after treatment (Figure 1, panel D). Corneal fibroblasts treated with poly I:C increased the expression of intracellular receptors TLR-3 and TLR-7 suggesting that poly I:C and MDP lead preferably to the expression of intracellular TLRs in these cells.

To explain how PGN could induce the expression of almost all TLRs, we performed in silico TLRs promoter regions analysis, looking for NF- $\mathrm{BB}$, interferon regulatory factor (IRF) and c-Jun recognition sites (Table 1). NF- $\kappa \mathrm{B}$ and c-Jun recognition sequences were found in almost all TLR promoters. NF- $\kappa B$ recognition sequences were absent in the TLR-9 promoter. This could explain why PGN can activate NF- $\mathrm{KB}$ and induce the overexpression of the majority of TLRs mRNAs, except TLR-9. Although other experiments should be done to support experimentally this suggestion, it has been already shown that TLR-2 and TLR-4 promoters have NF- $\kappa B$ recognition sites and that TLR-2 and TLR-4 are over-expressed through NF- $\mathrm{KB}(6)$, activator protein 1 (AP1) (2), PU.1 (2) and Sp1 (12) activation. There is no information about the transcription factors that are involved in the other TLRs. c-Jun recognition sequences were absent in the TLR-1 promoter and IRF recognition sequences were found with less frequency than NF- $\mathrm{KB}$, and it is only present in TLR-2 and TLR-5 promoters. According to the above mentioned, we suggest that NF- $\mathrm{KB}$ could be the main transcription factor involved in the TLRs induction of expression (or over-expression) in PGN stimulated cells.

In summary, this work shows that PGN from $S$. aureus is a potent activator for the expression of several TLRs mRNAs in corneal fibroblasts, meanwhile MDP and poly I:C induce preferentially intracellular TLRs mRNAs.

Table 1. NF- $\mathrm{KB}$, IRF and c-Jun binding-sites in TLRs promoters. These sites were found analyzing in silico the TLRs promoter regions (2000pb up-stream) using AliBaba 2.1, TESS and JASPAR softwares.

\begin{tabular}{|c|c|c|c|c|c|c|}
\hline TLR & Number of NF-KB sites & Position in promoter & Number of IRF1 sites & Position in promoter & Number of c-Jun sites & Position in promoter \\
\hline \multirow[t]{2}{*}{ TLR1 } & 2 & $268-277$ & 0 & 0 & 0 & 0 \\
\hline & & $546-555$ & & & & \\
\hline \multirow[t]{3}{*}{ TLR2 } & 3 & $342-351$ & 1 & $1036-1045$ & 1 & $762-771$ \\
\hline & & $1033-1042$ & & & & \\
\hline & & $1988-1997$ & & & & \\
\hline \multirow[t]{2}{*}{ TLR3 } & 2 & $387-396$ & 0 & 0 & 1 & $259-268$ \\
\hline & & $1949-1958$ & & & & \\
\hline \multirow[t]{3}{*}{ TLR4 } & 1 & $348-357$ & 0 & 0 & 3 & $15-24$ \\
\hline & & & & & & $134-143$ \\
\hline & & & & & & $659-668$ \\
\hline TLR5 & 1 & $1102-1111$ & 1 & $369-378$ & 1 & $344-353$ \\
\hline TLR6 & 1 & 1986-1995 & 0 & 0 & 1 & $70-79$ \\
\hline TLR7 & 1 & 1983-1992 & 0 & 0 & 1 & $1243-1252$ \\
\hline TLR8 & 1 & $937-946$ & 0 & 0 & 1 & $1702-1711$ \\
\hline \multirow[t]{2}{*}{ TLR9 } & 0 & ---------- & 0 & 0 & 2 & $1177-1186$ \\
\hline & & & & & & $1938-1950$ \\
\hline
\end{tabular}

* To consider the recognition sites as real, it was requested that the sites were found in the three programs. 


\section{ACKNOWLEDGEMENTS}

This work was supported by CONACYT grant 47424 . Mario E. Cancino-Díaz, Sandra Rodriguez-Martinez and Juan C. Cancino-Diaz are fellows of COFAA and EDI (IPN) and SNI (CONACYT)

\section{REFERENCES}

1. Akira, S.; Uematsu, S.; Takeuchi, O. (2006). Pathogen recognition and innate immunity. Cell. 124 (4), 783-801.

2. Bondeva, T.; Roger, T.; Wolf, G. (2007). Differential regulation of Tolllike receptor 4 gene expression in renal cells by angiotensin II: dependency on AP1 and PU.1 transcriptional sites. Am. J. Nephrol. 27 (3), 308-314.

3. Dziarski, R.; Gupta, D. (2006). Mammalian PGRPs: novel antibacterial proteins. Cell Microbiol. 8 (7), 1059-1069.

4. Faure, E.; Thomas, L; Xu, H.; Medvedev, A. E.; Equils, O.; Arditi, M. (2001). Bacterial lipopolysaccharide and IFN- $\gamma$ induce toll-like receptor 2 and toll-like receptor 4 expression in human endothelial cells: Role of NF- $\kappa$ B activation. J. Immunol. 166 (3), 2018-2024.

5. Heyer, G.; Saba, S.; Adamo, R.; Rush, W.; Soong, G.; Cheung, A.; Prince, A. (2002). Staphylococcus aureus agr and sarA functions are required for invasive infection but not inflammatory responses in the lung. Infect. Immun. 70 (1), 127-133.

6. Johnson, C. M.; Tapping, R. I. (2007). Microbial products stimulate human Toll-like receptor 2 expression through histone modification surrounding a proximal NF-kappaB-binding site. J. Biol. Chem. 282 (43),
31197-31205.

7. Kumar, A.; Zhang, J.; Yu, F. S. (2004). Innate immune response of corneal epithelial cells to Staphylococcus aureus infection: role of peptidoglycan in stimulating proinflammatory cytokine secretion. Invest. Ophthalmol. Vis. Sci. 45 (10), 3513-3522.

8. Melmed, G.; Thomas, L. S.; Lee, N.; Tesfay, S. Y.; Lukasek, K.; Michelsen, K. S.; Zhou, Y.; Hu, B.; Arditi, M.; Abreu, M.T. (2003). Human intestinal epithelial cells are broadly unresponsive to Toll-like receptor 2-dependent bacterial ligands: implications for host-microbial interactions in the gut. J. Immunol. 170 (3), 1406-1415.

9. Otte, J. M.; Rosenberg, I. M.; Podolsky, D. K. (2003). Intestinal myofibroblasts in innate immune responses of the intestine. Gastroenterology. 124 (7), 1866-1878.

10. Rodríguez-Martínez, S.; Cancino-Díaz, M. E.; Jiménez-Zamudio, L.; García-Latorre, E.; Cancino-Díaz, J. C. (2005). TLRs and NODs mRNA expression pattern in healthy mouse eye. Br. J. Ophthalmol. 89 (7), $904-$ 910.

11. Wang, X.; Zhang, Z.; Louboutin, J. P.; Moser, C.; Weiner, D. J.; Wilson, J. M. (2003). Airway epithelia regulate expression of human betadefensin 2 through Toll-like receptor 2. FASEB J. 17 (12), 1727-1729.

12. Wasiluk, K. R.; McCulloch, K. A.; Banton, K. L.; Dunn, D. L. (2006). Sp1 elements regulate transcriptional activity within the murine Toll-like receptor 4 promoter. Surg. Infect. 7 (6), 489-499.

13. Wyllie, D.H.; Kiss-Toth, E.; Visintin, A.; Smith, S. C.; Boussouf, S.; Segal, D. M.; Duff, G. W.; Dower, S.K. (2000). Evidence for an accessory protein function for Toll-like receptor 1 in anti-bacterial responses. J. Immunol. 165 (12), 7125-7132.

14. You, L.; Kruse, F. E.; Bacher, S.; Schmitz, M. L. (2002). Lipoteichoic acid selectively induces the ERK signaling pathway in the cornea. Invest. Ophthalmol. Vis. Sci. 43 (7), 2272-2277. 